\title{
Efficacy and safety of the weight-loss drug rimonabant
}

\author{
Jean-Pierre Després ${ }^{1}$, Luc Van Gaal ${ }^{2}$, Xavier Pi-Sunyer ${ }^{3}$, André Scheen ${ }^{4}$ \\ ${ }^{1}$ Quebec Heart Institute, Laval Hospital Research Center, Laval University, Sainte-Foy, Quebec G1V 4G5, Canada ; ${ }^{2}$ Department of \\ Diabetology, Metabolism, and Clinical Nutrition, University Hospital Antwerp, Faculty of Medicine (Department of Diabetology, \\ Metabolism, and Clinical Nutrition), Edegem-Antwerp, Belgium ; ${ }^{3}$ St Luke's-Roosevelt Hospital Center, New York, NY, USA; ${ }^{4}$ Division of \\ Diabetes, Nutrition, and Metabolic Disorders, CHU Sart Tilman, University of Liege, Liege, Belgium.
}

Robin Christensen and colleagues' meta-analysis (Nov 17, p 1706) ${ }^{1}$ contains several inaccuracies that should be noted.

The assertion that all patients with depressed mood were excluded from the RIO studies is incorrect. The protocols specifically excluded only patients with "clinically significant psychiatric disease and history of severe depression that could be defined as depression leading the patient to be hospitalised, or patients with two or more recurrent episodes of depression, or a history of suicide attempt".

In figure 4, Christensen and colleagues' use of a combined composite endpoint for depressed-mood disorders for the RIO-Lipids study overestimates the true rates, since some patients might have had more than one depressive adverse event leading to treatment discontinuation.

Christensen and colleagues' caution that some adverse events might not have been documented is unwarranted. All sites were revisited retrospectively to assess whether discontinuations not specifically ascribed to adverse event(s) were actually associated with adverse events. The relative risks of withdrawal for adverse events were unchanged.

In discussing the US Food and Drug Administration Advisory Committee deliberations, Christensen and colleagues seem to confuse the terms "suicide" and "suicidality". Moreover, a higher rate of "suicidal ideation" has not clearly been shown to translate into a higher rate of suicide. ${ }^{2}$

The revised product label for rimonabant stresses the importance of selecting the appropriate patient population-ie, overweight or obese individuals with cardiometabolic risk factors and no major depressive illness or ongoing antidepressive treatment. ${ }^{3}$

Finally, Christensen and colleagues' assertion that "no follow-ups were reported after discontinuation of the active treatment,thus any weight regain could be not assessed" is incorrect. The weight regain issue was specifically addressed during the randomised double-blind withdrawal period in the second 12 months of the RIO-North America trial. Patients switching from rimonabant $5 \mathrm{mg}$ or $20 \mathrm{mg}$ to placebo regained weight to a level comparable with that of patients treated with nutritional recommendations alone for 2 years. Beneficial effects of rimonabant on bodyweight, waist circumference, HDL cholesterol, and triglycerides were maintained (in RIO-North America and RIO-Europe) during 2 years of continuous rimonabant treatment. These results show the absence of a "rebound" effect after discontinuation of rimonabant, and establish its long-term maintenance effect not only on bodyweight and waist but also on the cardiometabolic risk profile, and confirm the well established principle that, as with chronic diseases such as hypertension or diabetes, maintenance of efficacy requires treatment continuation.

\section{References}

1 Christensen R, Kristensen PK, Bartels EM, Bliddal H, Astrup A. Efficacy and safety of the weight-loss drug rimonabant: a meta-analysis of randomised trials. Lancet 2007; 370:1706-13.

2 Klein D.The flawed basis for FDA postmarketing decisions: the example of anti-depressants and children. Neuropsychophamiacology 2006; 31: 689-99.

3 European Medicines Agency. Acomplia: summary of Product Characteristics, http:// www.emea.europa.eu/humandocs/PDFs/ EPAR/acomplia/H-666-PI-en.pdf (accessed Nov 21, 2007). 
JPD is a speaker for Abbott Laboratories, AstraZeneca, Fournier Pharma/Solvay Pharma, GlaxoSmithKline, and Pfizer Canada; he is on the Advisory board of MSD, Sanofi-Aventis, and Novartis; he has received Research funding from Sanofi-Aventis and GlaxoSmithKline and consulting fees from Innodia and Sanofi-Aventis. LVG has received research grants from Fonds Wetenschappelijk Onderzoek, Vlaanderen, and from an EU consortium "Hepadip project"; is on the speaker bureau for Abbott Pharma, AstraZeneca, and Sanofi-Aventis; and is a consultant and member of advisory boards for Abbott Pharma, Amylin Pharma, Johnson \& Johnson, Pfizer, and Sanofi-Aventis. XPS is a consultant to Sanofi-Aventis. AJS is on the speaker bureau for Sanofi-Aventis, Pfizer, AstraZeneca, and GlaxoSmithKline, and is a consultant and member of advisory boards for Sanofi-Aventis, GlaxoSmithKline, AstraZeneca, Eli Lilly, Merck-Santé, and Novo Nordisk. 